\title{
GLOBAL MAPS FROM INTERFEROMETRIC TANDEM-X DATA: APPLICATIONS AND POTENTIALS
}

\author{
Paola Rizzoli, Michele Martone, Benjamin Bräutigam, and Manfred Zink \\ German Aerospace Center (DLR), Microwaves and Radar Institute, Oberpfaffenhofen, Germany
}

\begin{abstract}
TanDEM-X is a spaceborne Synthetic Aperture Radar (SAR) mission, whose goal is the generation of a global Digital Elevation Model (DEM) with unprecedented accuracy, by using interferometric SAR (InSAR) techniques (InSAR). TanDEM-X offers a huge global data set of bistatic InSAR acquisitions, each of them supplemented by quicklook images of different SAR quantities, such as amplitude, coherence, and DEM. Global quicklook mosaics of the interferometric coherence and of the relative height error can be considered for mission performance monitoring and acquisition strategy optimization. The aim of this paper is to present the use of such mosaics within the TanDEM-X mission and to show their potentials for future scientific applications for example in the fields of glaciology and forestry.
\end{abstract}

Key words: TanDEM-X; Interferometry; Forest Map; Greenland; Ice Classification.

\section{INTRODUCTION}

The primary objective of the TanDEM-X mission is the generation of a global, high-precision digital elevation model by using synthetic aperture radar interferometry (InSAR). The continuous monitoring of quality parameters is necessary for optimizing the acquisition strategy and achieving the final mission specification. TanDEM$X$ offers a vast global data set of multiple InSAR acquisitions, each of them supplemented by quicklook images of different SAR quantities, such as amplitude, coherence, and DEM.

One of the main parameters for assessing the quality of interferometric products is the interferometric coherence between the monostatic and the bistatic images [1]. Global mosaics of such a quantity are operationally used to quickly analyze the mission performance and to derive further key-quality parameters, such as the relative height error. Their analysis represents a powerful mean for optimizing the acquisition planning. For example, large-scale mosaics of the relative height error over sandy desert areas have allowed to determine critical regions to be reacquired using dedicated geometries, in order to improve the final DEM quality [7].

Moreover, large-scale mosaics of the interferometric coherence represent a valid starting point for further scientific applications and for the generation of add-on products from the TanDEM-X global data set.

In the field of glaciology, the classification of snow pack facies is an interesting and open topic. The combination of the information coming from both backscatter intensity and interferometric coherence can be exploited for better classifying different kinds of snow, dominated by different scattering mechanisms. An example is the definition of the snow facies of the Greenland ice-sheet, obtained by combining backscatter and decorrelation information.

The quality of interferometric data pairs is strongly influenced by the specific land-cover type of the illuminated scene on ground. Over vegetated areas, multiple scattering mechanisms within the same resolution cell lead to volume decorrelation. By exploiting such information, a global map of vegetated areas can be derived. The amount of volume decorrelation is also a good indicator of the vegetation density and sets the basis for the development of a forest-type classifier.

The aim of this paper is to present the described mosaics derived from TanDEM-X data, to explain their operational use within the TanDEM-X mission, and to introduce the scientific potential of the global TanDEM-X interferometric data set, not only for topography measurements, but for further scientific applications as well.

\section{GLOBAL MOSAICS FROM TANDEM-X QUICKLOOKS}

For data handling reasons within the nominal TanDEM-X processing chain, long acquisitions are split into scenes of about $50 \mathrm{~km}$ azimuth length, and with swath widths of about $30 \mathrm{~km}$. By January 2015, about 500,000 bistatic scenes have been processed by the operational Integrated TanDEM-X Processor (ITP) [2], showing that TanDEM-X is able to provide the remote sensing community a unique data set to be exploited for a wide range of scientific applications. For each TanDEM-X bistatic scene, the ITP produces several quicklooks as by-products from the interferometric processing chain. These quicklooks are characterized by a lower resolu- 
tion compared to the corresponding interferometric data at full resolution The ground pixel spacing is of about $25 \times 25 \mathrm{~m}^{2}$ (whereas the final, and are taken into account as input data base for generating quicklook mosaics. The quicklook mosaics presented in this work are generated by bilinearly interpolating images into a common output grid, as explained in [3]. The output pixel spacing can be selected to resolutions down to $25 \times 25 \mathrm{~m}^{2}$. For global monitoring purposes, and because of data handling reasons, we have chosen an output pixel spacing of $0.003^{\circ}$, corresponding to about $300 \times 300 \mathrm{~m}^{2}$ at the equator.

\section{PERFORMANCE MONITORING AND ACQUISITION PLANNING SUPPORT}

In this section we focus on the use of global quicklook mosaics for interferometric performance monitoring purposes. In particular, we describe the monitoring and optimization of the relative height error within the DEM, which is a random error related to decorrelation sources affecting the interferometric data [4]. The fundamental parameter for evaluating the relative height error from the interferometric data is the interferometric coherence, allowing to derive the relative height error standard deviation as described in [1]. A global quicklook mosaic of the interferometric coherence, derived from TanDEM-X data acquired during the first year of operation, is presented in Fig. 1. The relative height error performance is influenced by several factors, such as the height of ambiguity and the type of land cover on ground. The height of ambiguity is the physical height corresponding to a $2 \pi$ difference within the interferogram and is baseline dependent. Such quantity has been regularly monitored during all different coverages of the TanDEM-X mission, in particular: first year global coverage with height of ambiguity between $40 \mathrm{~m}$ and $55 \mathrm{~m}$, second year global coverage with height of ambiguity between $30 \mathrm{~m}$ and $40 \mathrm{~m}$ and displaced elevation beams in order to guarantee an almost constant performance in range, additional coverage with dedicated acquisitions over critical areas (typically forested and mountainous areas) to improve the interferometric coherence using higher height of ambiguity, desert acquisitions over desert areas with steeper incidence angles, and crossing orbit acquisitions over mountainous terrain to solve shadow and layover issues [6]. Particularly relevant is the monitoring of critical areas, where the acquisition strategy has to be optimized in order to obtain a final performance within the mission specifications (90\% relative height error confidence level within tiles of $1^{\circ} \times 1^{\circ}$ in latitude/longitude coordinates lower than $2 \mathrm{~m}$ for low-relief terrain and lower than $4 \mathrm{~m}$ for high-relief terrain). For example, mountainous regions, which are identified as areas with terrain slope higher than $20 \%$, are typically affected by geometric distortion phenomena, such as shadow and layover. Within the TanDEM$\mathrm{X}$ mission, the northern hemisphere has been nominally acquired in ascending orbit direction, while the southern one in descending orbit direction. If a ground region is always illuminated using the same orbit direction, some ar- eas might remain in shadow or layover in all acquisitions, leading to unreliable height estimations within the final DEM. The acquisition of mountainous regions has been performed on a global scale with opposite viewing direction (crossing orbit), allowing to improve the final relative height error performance and to minimize the number of void pixels. An example over the Alps region is presented in Fig. 2: the quicklook mosaic of the the relative height error standard deviation, obtained from the combination of first and second year acquisitions, acquired in ascending orbit direction, is presented in Fig. 2(a), while the one from crossing orbit acquisitions, performed in descending orbit direction, is depicted in Fig. 2(b). The combination of both ascending and descending orbit acquisitions is shown in Fig. 2(c). The achieved confidence levels for $4 \mathrm{~m}$ over high-relief terrains are $93.93 \%$, $85.1 \%$, and $98.41 \%$, respectively. Moreover, a zoom of the small area inside the black square better underlines such a performance improvement.

Other critical areas are sandy deserts, characterized by low backscatter. In this case, a dedicated re-acquisition strategy using steeper incidence angles has been executed [7]. The generation of mosaics on a global scale is highly important for monitoring the whole TanDEM-X mission performance. A global map of the relative height error confidence level for $1^{\circ} \times 1^{\circ}$ tiles, derived from a quicklook mosaic of the relative height error standard generated by composing all the available TanDEM-X data from all coverages up to September 2014, is depicted in Fig. 3. Low- and high-relief terrain are properly distinguished by applying a threshold at $20 \%$ on a slope map, which is derived by evaluating the bidimensional gradient of the corresponding TanDEM-X quicklook DEM, as presented in [4] (84.4\% of the covered land is characterized by lowrelief terrain). The required point-to-point relative height error confidence level is then derived from these two distributions as presented in [5]. As it can be seen, the relative height error specification is achieved for more than $90 \%$ of all the available tiles. Highly vegetated and snowcovered tiles which do not achieve the TanDEM-X specification are identified in gray. Such tiles are not taken into account for the evaluation of the global mission performance.

\section{DISCRIMINATION OF THE GREENLAND ICE SHEET FACIES}

The Greenland ice sheet is a vast ice-covered plateau extending over $80 \%$ of the whole Greenland surface. Melting phenomena have strongly increased in the last years, leading to changes in the characteristics of the snow pack. Previous studies of the Greenland ice sheet led to the definition of different snow facies, depending on the amount of melting and on the properties of the snow coverage itself. C. S. Benson divided the ice sheet into four zones [8]: melting does not occur in the inner dry snow zone, which is situated at the highest altitudes at the center of the Greenland plateau. It is surrounded by the so called percolation zone, where a limited amount of melting occurs, leading to the generation of larger snow grains and 


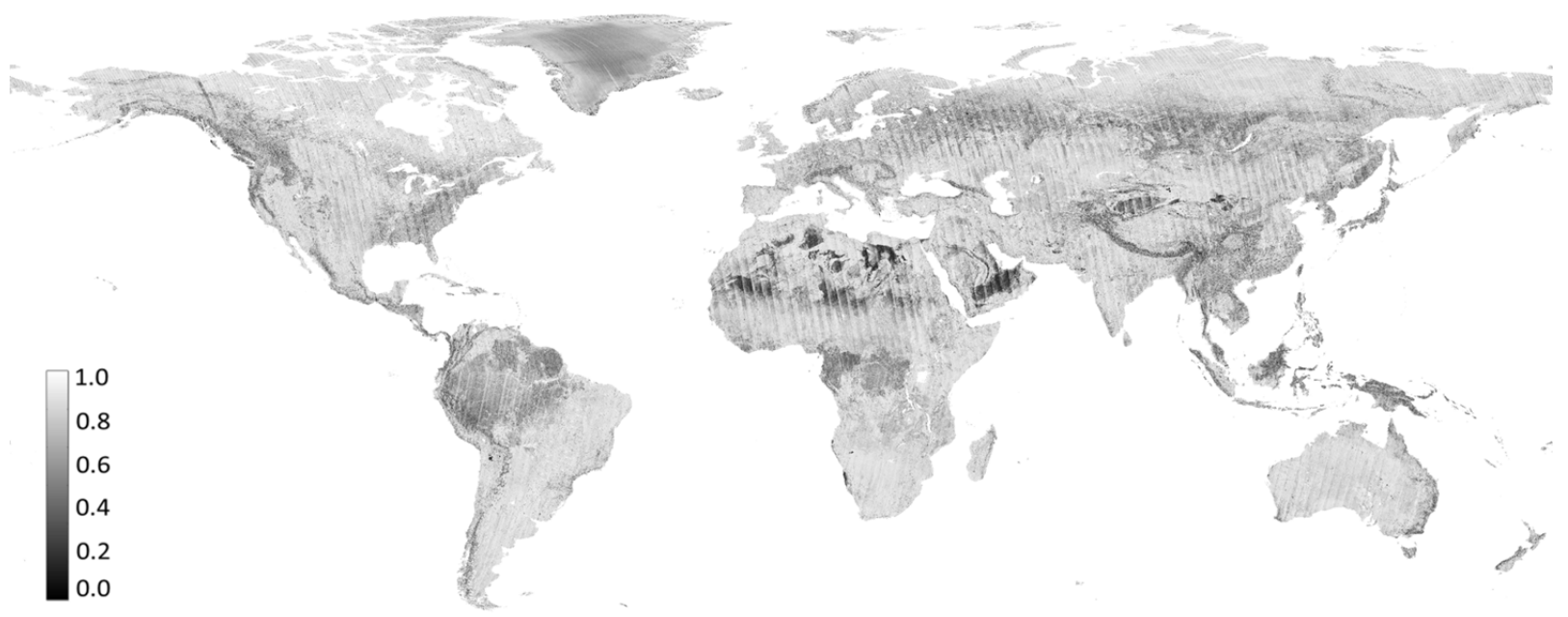

Figure 1: Global map of the interferometric coherence, based on the TanDEM-X first coverage acquisitions. Water has been filtered out using a water mask, obtained from the GLOBCOVER classification map.

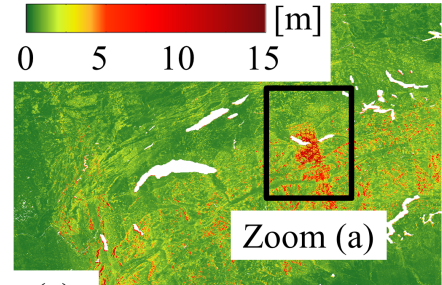

(a)
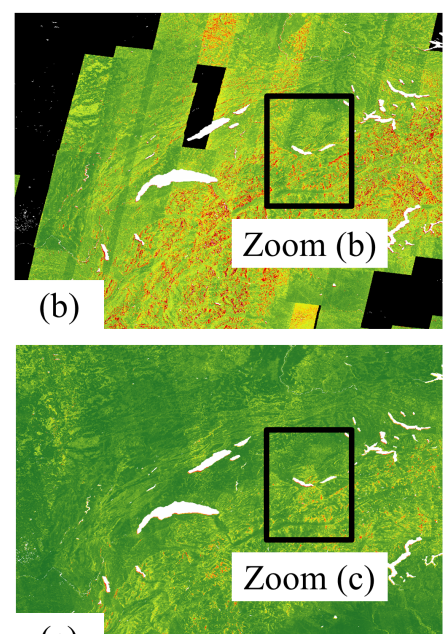

(c)

Figure 2: Mosaics of the relative height error standard deviation over the Alps for different TanDEM-X coverages.

to the formation of small ice structures, such as lenses and cones, within the snow pack. The wet snow zone is located further down slope towards Greenland's coasts, where previous year accumulation saturates with water during summer melt. Outer coastal regions are classified as ablation zone, where the previous year accumulation completely melts during summer, resulting in a sur- face of bare ice and rocks. Up to now, such facies have been located using microwaves radar sensors by estimating the backscatter levels of the reflected signal [9]. An incident radar wave is able to penetrate the snow-pack, being therefore subjected to volume decorrelation. The intensity of such phenomena can be associated to different dominant scattering mechanisms on ground and can therefore help to classify the type of snow coverage as well.

The basic idea for locating the different facies of the Greenland ice sheet is to exploit the information available from interferometric acquisitions and, in particular, to combine volume decorrelation intensity and radar backscatter. Large-scale mosaics over Greenland of such quantities represent therefore the starting input for the developed technique. Moreover, the interferometric coherence is influenced by the height of ambiguity. In order to generate a mosaic, images, acquired with different heights of ambiguity have to be combined together. Therefore, a correction factor has been evaluated by performing a linear fitting on the each mean coherence per scene acquired over Greenland, with respect to to the corresponding height of ambiguity. Its application allows to refer all images to a reference height of ambiguity and mitigate therefore the effects such a dependency. The volume decorrelation can then be directly derived from the interferometric coherence by compensating for the remaining decorrelation sources, such a limited SNR or quantization noise, already presented in [1]. The mosaics of volume decorrelation and backscatter, derived by composing TanDEM-X scenes acquired during winter 2011, are presented in Fig. 4.

For classifying the ice sheet, we decided to apply fuzzy clustering techniques, aiming at grouping together elements coming from an input set of observations, depending on how similar they are to each other. Such observations are then divided into $c$ non-empty subsets called clusters. Fuzzy clustering allows different clusters to overlap and assigns to each observation a membership 


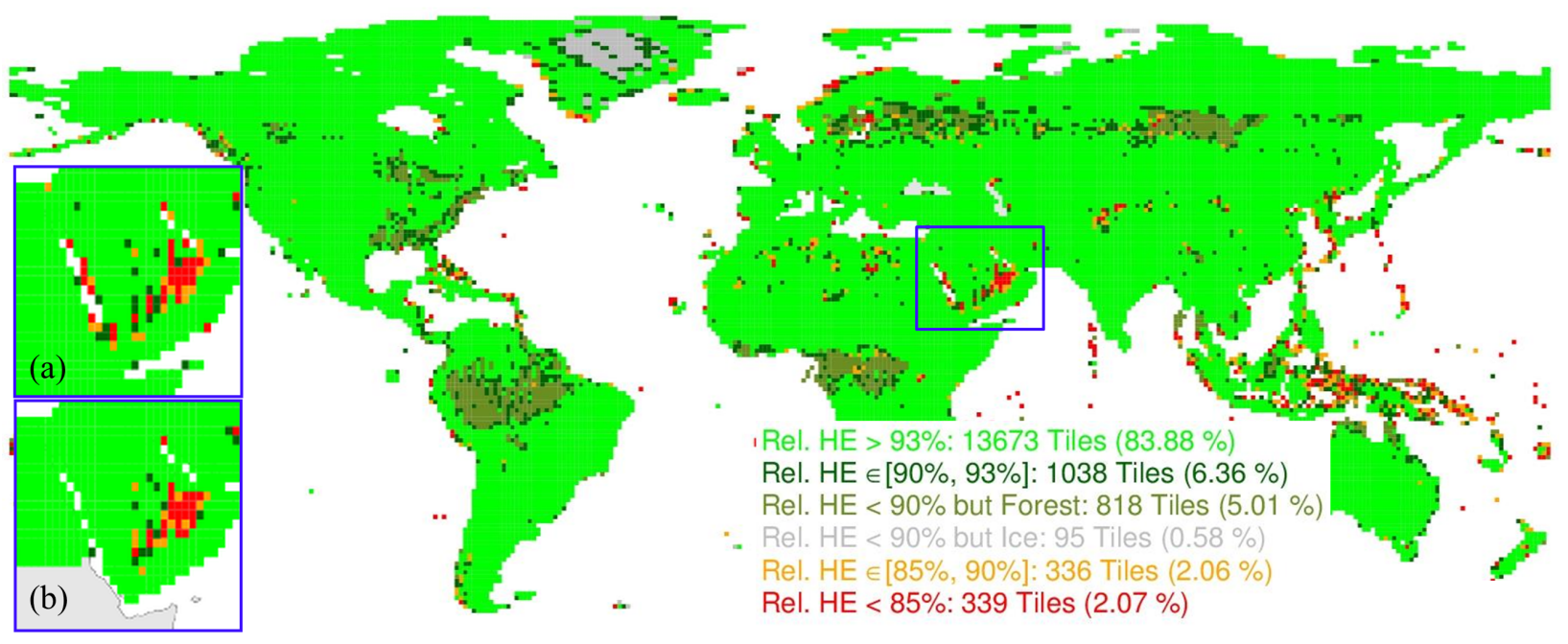

Figure 3: estimated confidence level of the relative height error per tile, obtained by composing acquisitions from all the available TanDEM-X coverages (status September 2014).

value for each cluster, which represents the probability of the observation to belong to it. The fuzzy $c$-Means clustering algorithm has been used to determine the clusters' centers [10], defined as the mean of all observations, weighted by their degree of belonging to the cluster. The clusters centers are derived by locally minimizing an objective function, given in this case by the Euclidean norm between the cluster centers and the observations. The membership of an observation to a specific cluster is inversely related to such a distance. Namely, two features have been associated to each observation: volume decorrelation and backscatter. Each cluster center is then a bi-dimensional vector, where each element represents the cluster center for a single feature. We chose to initialize the algorithm using four different clusters. The resulting membership values for each cluster after convergence is obtained are displayed in Fig. 5. As it can be seen, each cluster is characterized by a different distribution on the ice sheet and four different regions are detectable. The classification can then be performed by selecting the highest membership value per pixel. The result is shown in Fig. 6. The ice sheet borders are clearly delimited by an extended region identified in green, which can be associated to Benson's percolation and then wet snow zones. In this way the outer coastal areas can be separated from the ice sheet. After a transition zone, identified in blue, the most inner region of the plateau is characterized by the presence of dry snow, compatible to the extension of the red and orange areas. An explication of the presence of two different clusters within the same dry snow facies can be given by the different snow accumulation rate: the inner part the plateau is characterized by higher altitude and accumulation rate. Moreover, the grain size is typically smaller, which results in a higher absorption of the radar wave and consequently in a lower intensity of volume scattering. Up to now, this approach has been implemented by supposing a fixed number of clusters, in order to start exploring the potentials of the interferometric data set. Given the encouraging results, the future steps

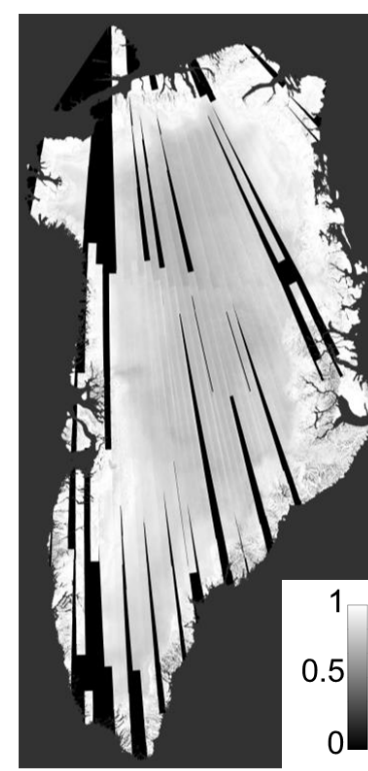

Volume Decorrelation

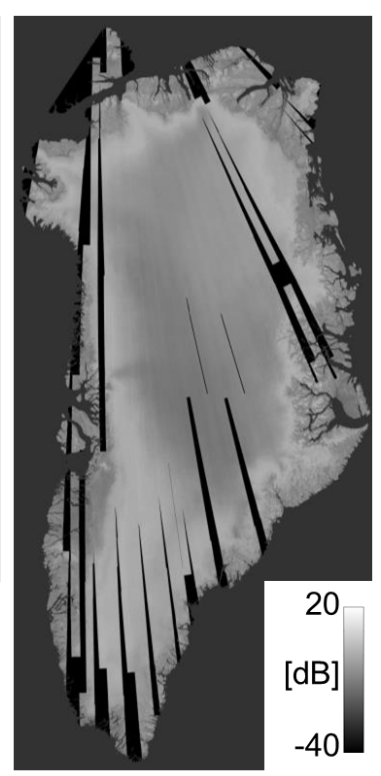

$\operatorname{Backscatter}\left(\gamma^{0}\right)$
Figure 4: Left: mosaic over Greenland of volume decorrelation from TanDEM-X images acquired during winter 2011. Right: corresponding backscatter mosaic $\left(\gamma^{0}\right)$.

will be to find the optimal number of clusters and their centers, by exploiting quality indicators on the compactness and separation of the observations belonging to a single cluster. Finally, the obtained results will have to be verified with external data. 

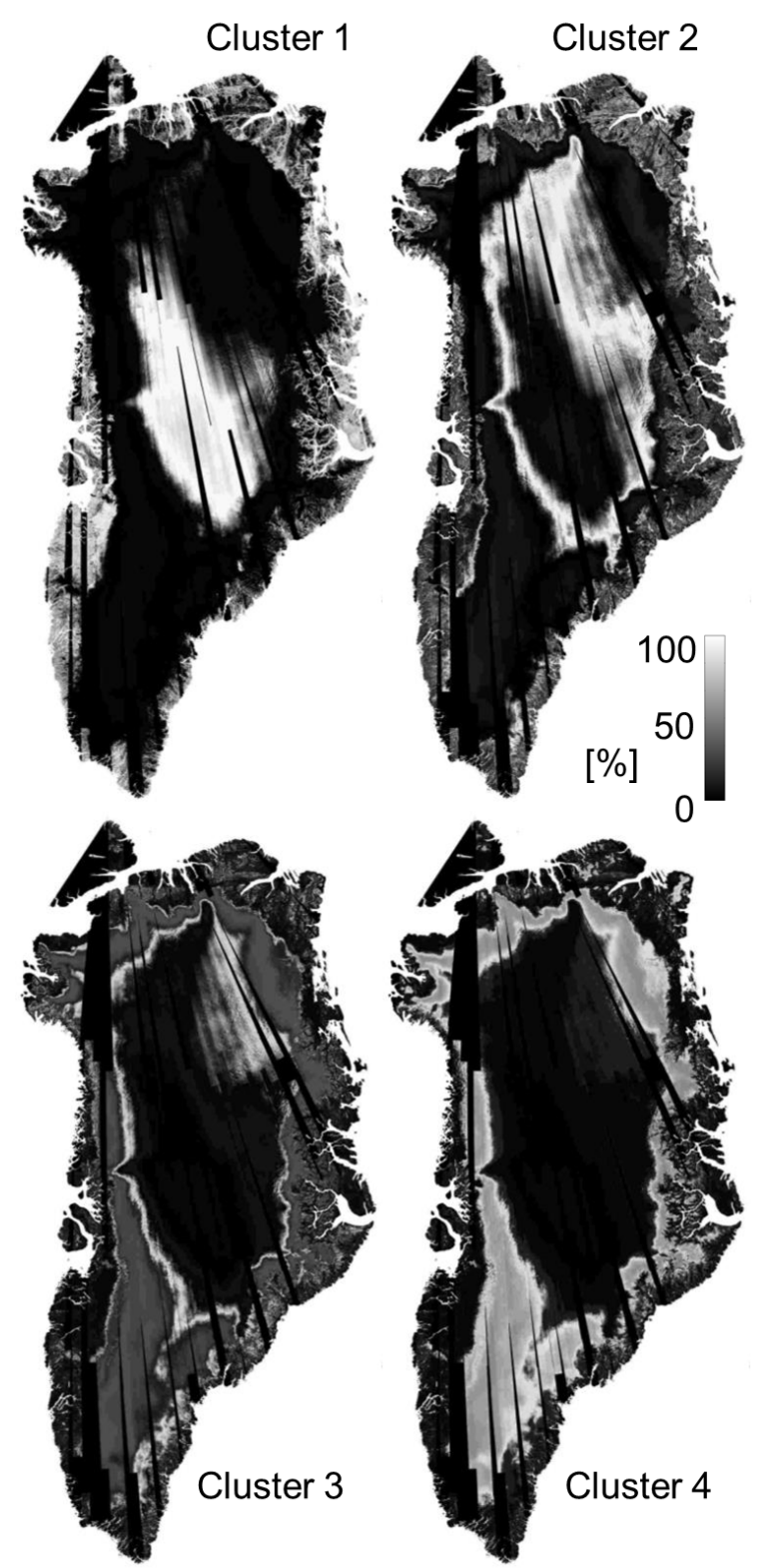

Figure 5: Membership values for observations over the Greenland Ice Sheet, obtained by applying the fuzzy $c$ Means clustering algorithms with four clusters.

\section{FOREST/NON-FOREST MAP}

Land-cover classification by means of remote sensing data is of fundamental importance for a broad range of commercial and scientific uses. In particular, the detection of vegetated areas is of great interest for global change research and for applications in agriculture, cartography, geology, forestry, as well as regional planning. Over forested areas the presence of multiple scatterers at different heights and within a single resolution cell results in an increase of the interferometric phase uncertainty, leading to a volume decorrelation component, whose intensity depends on several factors, such as the acquisition

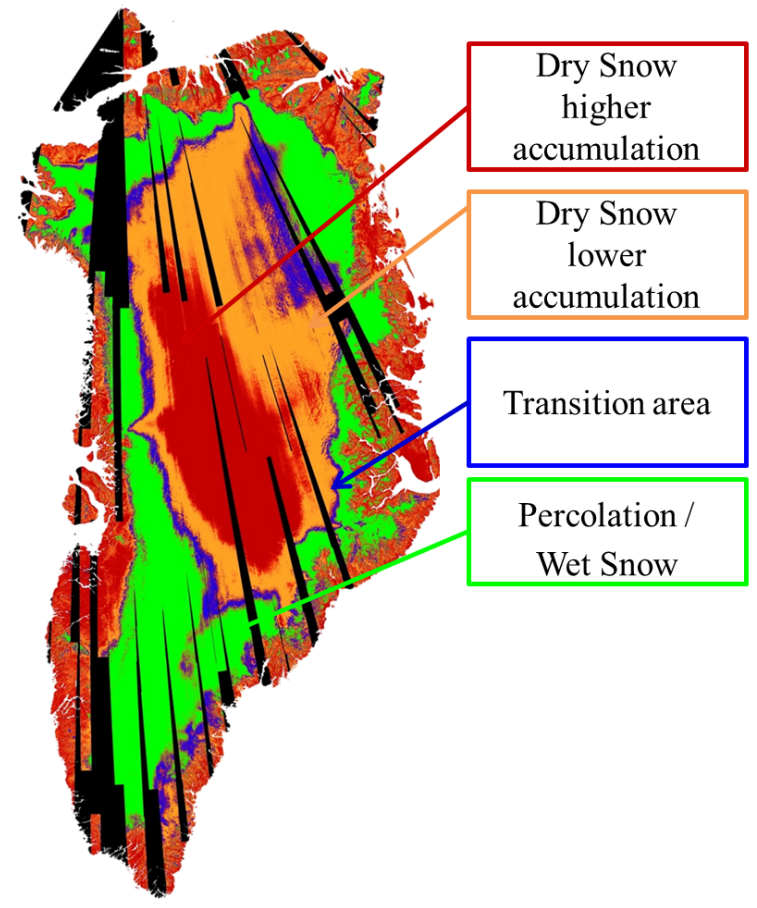

Figure 6: Classification map of the Greenland Ice Sheet, obtained by selecting the most likely cluster from the memberships in Fig. 5.

geometry, the sensor parameters, and the canopy density. Hence, from each bistatic TanDEM-X coherence map the volume decorrelation contribution can be estimated, in the same way as presented in sec. 4. A series of adaptive thresholds can then be applied in order to quantify the intensity of volume decorrelation in a limited number of intervals. An example is proposed in Fig. 7, where a TanDEM-X bistatic acquisition over Russia is considered. The volume decorrelation contribution is depicted in Subfig. (a), while a quantized version using three different levels is shown in Subfig. (b), where green, yellow, and red correspond to high, medium, and low volume decorrelation intensities, respectively. The further combination of multiple coverages leads to the generation of a forest/non-forest map, as presented in Fig. 8. Since vegetation density influences the intensity of volume decorrelation, different kinds of vegetation can be discriminated by properly setting a series of adaptive thresholds on the volume decorrelation images to be mosaicked. Another approach which is under study for the generation of forest/non-forest map is the use of fuzzy clustering techniques, as previously presented in sec. 4 for the localization of Greenland ice sheet facies.

\section{CONCLUSION}

In this paper we have described some of the potentials of the global interferometric TanDEM-X dataset. Large scale mosaics of several quantities can be generated from quicklook images. They are operationally used within the 


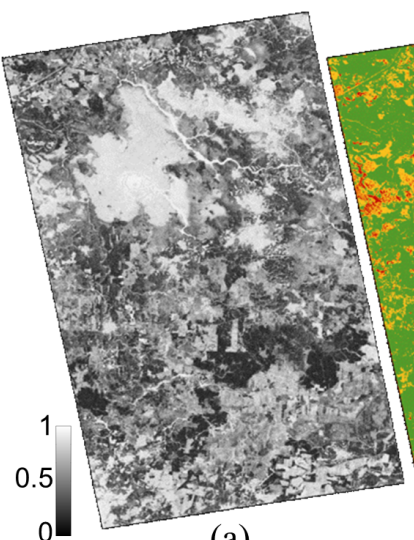

(a)

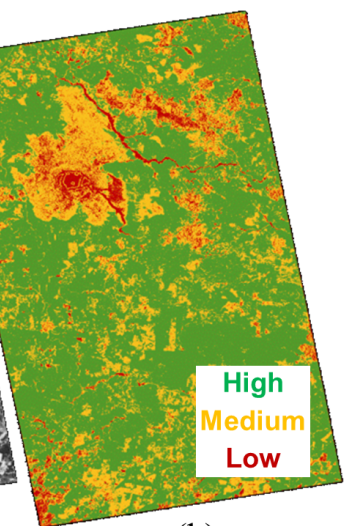

(b)
Figure 7: (a) - Volume decorrelation from a TanDEM$\mathrm{X}$ scene acquired over Russia. (b) - quantized volume decorrelation map, derived using three different levels: green - high, yellow - medium, red - low.

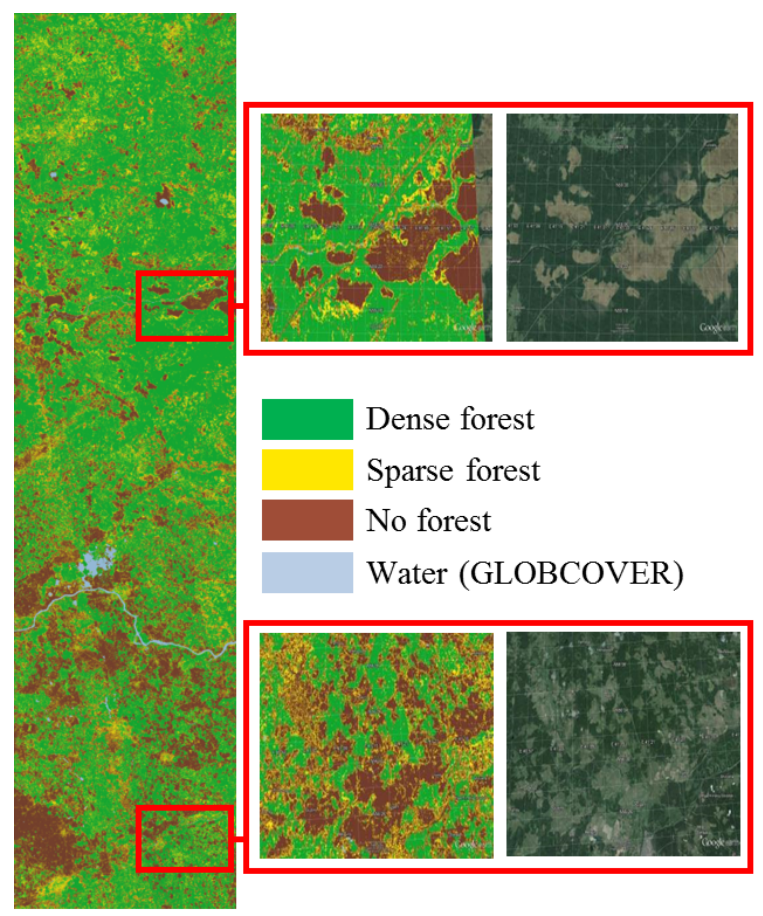

Figure 8: Forest/non-forest map obtained by combining multiple TanDEM-X coverages acquired over Russia, compared to optical images from Googleearth.

TanDEM-X mission for performance monitoring and optimization purposes. In particular, we presented the estimated final performance of the relative height error of the TanDEM-X DEM, confirming the outstanding capabilities of this unique single-pass spaceborne interferometer. We also introduced the use of the TanDEM-X dataset for global scientific purposes, in particular for snow/ice classification and forest detection. Further steps need to be investigated in this fields, but the acquired data shows to be a valid starting point for scientific uses.

\section{ACKNOWLEDGMENTS}

The TanDEM-X project is partly funded by the German Federal Ministry for Economics and Technology (Förderkennzeichen $50 \mathrm{EE} \mathrm{1035)}$ and realized in a publicprivate partnership between German Aerospace Center (DLR) and AIRBUS Defence \& Space.

\section{REFERENCES}

[1] G. Krieger; A. Moreira; H. Fiedler; I. Hajnsek; M. Werner; M. Younis; M. Zink: TanDEM-X: A Satellite Formation for High-Resolution SAR Interferometry, IEEE Transactions on Geos. and Remote Sens., vol. 45, no. 11, pp. 3317-3341, Nov. 2007.

[2] T. Fritz; C. Rossi; N. Yague-Martinez; F. RodriguezGonzalez; M. Lachaise; H. Breit: Interferometric Processing of TanDEM-X data, Geoscience and Remote Sensing Symposium (IGARSS) Proceedings, 24-29 July 2011, Vancouver (Canada).

[3] P. Rizzoli; M. Martone; B. Bräutigam: Global Interferometric Coherence Maps From TanDEM-X Quicklook Data, IEEE Geoscience and Remote Sensing Letters, vol. 11, no. 11, November 2014.

[4] P. Rizzoli; B. Bräutigam; T. Kraus; M. Martone; G. Krieger: Relative Height Error Analysis of TanDEM-X Elevation Data, ISPRS Journal of Photogrammetry and Remote Sensing, vol. 73, September 2012, pp. 3038 .

[5] C. Gonzalez; B. Brräutigam; M. Martone; P. Rizzoli: Relative Height Error Estimation Method for TanDEM-X DEM Products, $10^{\text {th }}$ European Conference on Synthetic Aperture Radar, Berlin, Germany, 2014.

[6] Borla Tridon D.; Bachmann M.; Schulze D,; OrtegaMiguez C.; Polimeni M. D.; Martone M.; Böer J.; Zink M.: TanDEM-X: DEM Acquisition in the Third Year, International Journal of Space Science and Engineering , 2014.

[7] M. Martone; B. Bräutigam; P. Rizzoli; G. Krieger: TanDEM-X Performance over Sandy Areas $10^{\text {th }}$ European Conference on Synthetic Aperture Radar, Berlin, Germany, Jun. 2014.

[8] C. S. Benson: Stratigraphic Studies in the Snow and Firn of the Greenland Ice Sheet, U.S. Army Snow, Ice and Permafrost Research Establishment, Research Report 70, Jul. 1962 (Reprinted Aug. 1996).

[9] E. Rignot; K. Jezek; J.J. van Zyl and M.R. Drinkwater: Radar scattering from snow facies of the Greenland ice sheet: results from the AIRSAR 1991 campaign, Geoscience and Remote Sensing Symposium, vol. 3, pag. 1270-1272, 18-21 Aug. 1993, Tokyo (JP).

[10] J. Bezdek; R. Ehrlich; W. Full: FCM: The Fuzzy c-Means Clustering Algorithm, Computers and Geosciences, col. 10, no. 2-3, pag. 191-203, 1984. 